EPJ Web of Conferences 66, 01009 (2014)

DOI: $10.1051 /$ epjconf/ 20146601009

(C) Owned by the authors, published by EDP Sciences, 2014

\title{
Recent progress in EDF-based methods applied to nuclear prop- erties
}

\author{
E. Khan ${ }^{1, a}$ \\ ${ }^{1}$ Institut de Physique Nucléaire, Université Paris-Sud, IN2P3-CNRS, F-91406 Orsay Cedex, France
}

\begin{abstract}
Recent results obtained with energy density functional (EDF) based methods are presented, focused on the nuclear phenomenology. EDF approaches aim for an universal description of the nuclear phenomena over the nuclear chart. Achievements from large to small nuclear systems are depicted: equation of state of nuclear matter, heavy and light nuclei. Dynamical aspects such as nuclear excitations, decay and reactions are also presented. EDF methods are progressing towards an unified and systematic description of the rich variety of the nuclear phenomena such as quantum liquid and cluster states, or nuclear structure and reactions.
\end{abstract}

\section{Introduction}

Energy Density Functional (EDF) based methods have accomplished dramatic progress during last decades. Dealing with the many-body problem, EDF methods are rooted in the nuclear density functional theory, where the word theory should be emphasized. It allows for a global description of the various nuclear phenomena from first principles, following the Hohenberg-Kohn theorem and the Kohn-Sham approach applied to nuclear systems [1-3]. EDF methods provide an intuitive picture of the nucleus and can be applied to all nuclei along the periodic chart, which is relevant to extrapolate predictions far from stability. Over the last few years, further significant progress have been accomplished using EDF methods. The present report focuses on recent achievements accomplished in this area with respect to the nucleus' phenomenology. Encompassing the large variety of the nuclear phenomenon is especially challenging. Recent progress made by EDF are going along this path. It should be noted that technical developments are also of interest and will be mentioned in the conclusion.

Various nuclear phenomenon have to be described: the quantum liquid behavior of the nucleus as well as the clusters states are maybe the most prominent features in nuclei. The interplay with the nuclear equation of state for possible astrophysical applications is also of relevance. To get a global and unified picture of the nucleus, EDF methods seem therefore to be well adapted. The formalism of EDF shall not be recalled here, and can be found in many reviews [4, 5]. Schematically, these approaches rely on a density functional, which is mainly of Skyrme, Gogny or relativistic type. Given this functional, which typically encompasses a dozen of constants, the many body treatment is parameter free and only technical refinements are allowed in the many body framework. It should be noted that the EDF constants are usually determined by fitting nuclear masses or radii and therefore the EDF includes in this way effective many body correlations, as illustrated by the accurate prediction

ae-mail: khan@ipno.in2p3.fr

This is an Open Access article distributed under the terms of the Creative Commons Attribution License 2.0, which permits unrestricted use, distribution, and reproduction in any medium, provided the original work is properly cited. 
of many nuclear binding energies. These effective constants are rather a strength than a weakness of the method. It is however difficult to exactly know which correlations are included or not through this mechanism.

EDFs allow to investigate the nuclear phenomenon in a unified framework from very heavy systems to light ones: from neutron stars and infinite nuclear matter, through heavy nuclei to light ones. Recent progress allow to initiate such a global view of the nuclear landscape. Not only nuclear structure but also nuclear dynamics can be described, such as excited states, nuclear decay and reaction predictions.

\section{Neutron star and infinite matter}

Nuclear matter is not only a toy imagined by theoreticians. It shall exist in the Universe, namely in neutron stars $[6,7]$. For instance the mass-radius prediction of such as star crucially depends on the nuclear Equation of State (EoS), and therefore on the EDF used. It can be shown that the nuclear incompressibility has an impact on this mass-radius relationship [6].

This raises the question of which density of nuclear matter is probed by nucleus' observables: is it the saturation density? Obviously not, since surface effects plays a role. More precisely, even in a heavy nucleus such as ${ }^{208} \mathrm{~Pb}$, two thirds of the nucleons are located in the surface of the nucleus, at a density below the saturation one. Consequently, the measurement of the compressibility mode in a nucleus (the Giant Monopole Resonance) constrains nuclear matter at a density below the saturation one. Such investigations are on their way [8]. The impact of subsaturation densities in EoS has also been noticed on other observables such as the neutron skin [9].

Subsaturation densities are therefore playing a role in constraining the EoS. What happens if the density of the nuclear system drops to very low values ? A clue is given in [11] where the data obtained from heavy ion collision at $35 \mathrm{MeV}$ per nucleon is well described by explicitly including clusterisation effects in the description. There are also indications that EDF methods naturally predict the transition from a quantum liquid system to a clusterised one when expanding it, that is diluting its density $[12,13]$. Therefore clusterisation naturally occurs in low density nuclear matter and this effect is encoded into EDFs. This path will be further investigated below in finite light nuclei.

More generally the constraints from the nuclear matter allow to validate EDFs. It has been recently shown that among the 240 Skyrme EDFs, only a dozen pass the systematic test on validated ranges of incompressibility, symmetry energy, effective masses, or describing two solar masses neutrons stars through the EoS [10]. This work paves the way towards defining sound criteria to validate EDFs to be used in further calculations.

\section{Heavy nuclei and quantum liquid}

Going towards smaller nuclear systems than infinite matter, nuclei, especially heavy ones, are known to exhibit quantum liquid features. The large mean free path (m.f.p.) of nucleons is an essential feature of the nucleus. It has recently been described using Green functions formalism, in agreement with the data, exhibiting a m.f.p. of about $5 \mathrm{fm}$ [14]. As discussed by Mottelson, the concept of independent particle motion is based on the fact that the orbits of individual nucleons are delocalised and reflects the shape and the radial dependence over the entire nucleus [15]. This is a typical quantum liquid behavior which is mostly visible through the large m.f.p. of the nucleons.

In the last decades, nuclear mass predictions using various EDFs hardly converged in their extrapolation far from stability [16]. Recent progress have been undertaken to estimate in a systematic way the errors (both statistical and systematic) associated to these functionals, allowing to predict $6900 \pm$ 
500 nuclei having $\mathrm{Z}<121$ in the nuclear chart [17]. This result, combined with the systematic selection of the EDFs mentioned above [10], paves the way to a sound delimitation of the nuclear landscape. It should be noted that EDFs have been recently designed to include data on deformed nuclei and/or nuclear excitation in the determination of their effective constants [18].

EDFs not only quantitatively describe the behavior of the liquid drop but are also able to predict qualitative new effects in nuclei. In a recent achievement including relativistic, superfluid, deformation and continuum effects in the description of ${ }^{42} \mathrm{Mg}$, an oblate neutron halo around a prolate neutron core was predicted [19] (Fig. 1). This is a typical motivating result for experimentalists, looking for new effects. Nuclear shapes can also be analysed. Recent works have been devoted to the rotation of slightly triaxial shapes in ${ }^{158} \operatorname{Er}[20]$. The prolate to oblate shape transition was also recently analysed in superheavy elements [21] and the good description of the data in ${ }^{190}$ Os using a mixed EDF-Interacting Boson Model approach showed that the triaxial shape is between a rigid triaxial and a $\gamma$ unstable shapes [22].
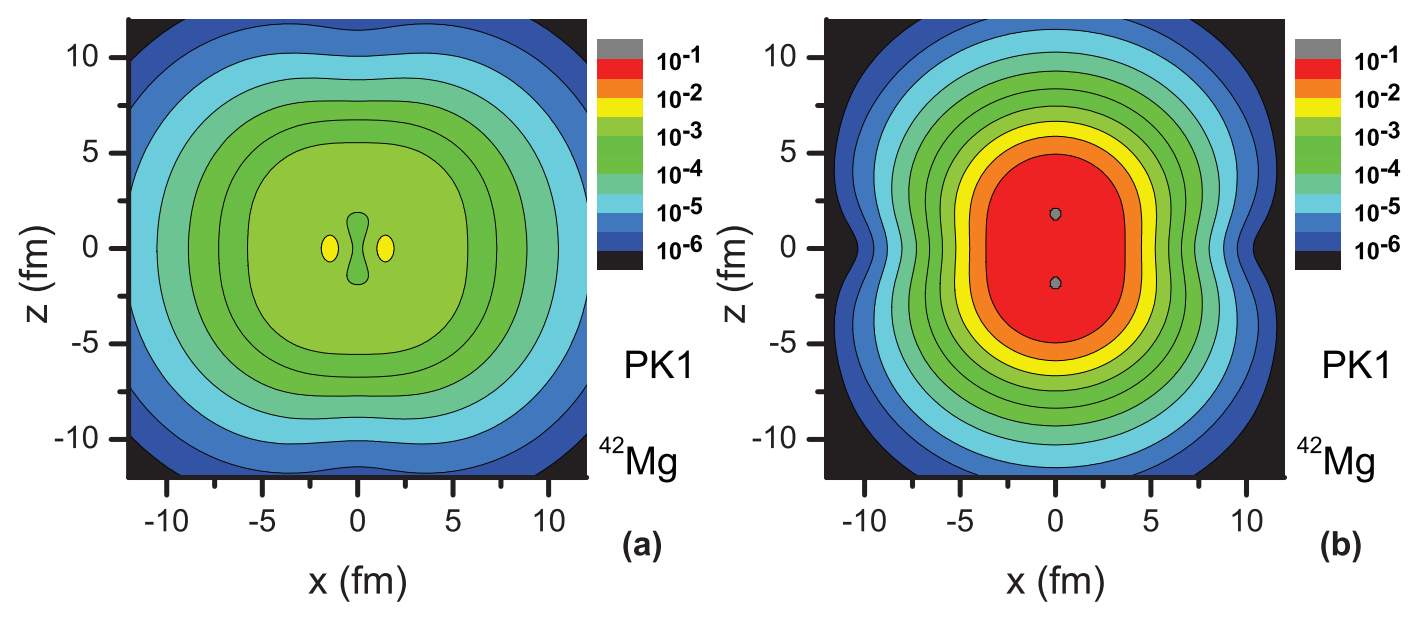

Figure 1. (Color online) Density distributions of the ground state of ${ }^{42} \mathrm{Mg}$ with the $\mathrm{z}$ axis as the symmetry axis: (a) neutron halo, and (b) neutron core. Reprinted figure with permission from L. Li et al., Phys. Rev. C 85, 024312 (2012). Copyright (2012) by the American Physical Society.

\section{Light nuclei and clusters}

Recent progress in EDF methods have been recently achieved to describe a important feature of lighter nuclei, namely the cluster states. This paves the way to a unified description of different nuclear states such as the quantum liquid and the cluster phenomena which coexist in nuclei. It should be noted that the following results are obtained with standard EDF approaches without any additional assumption on clusterisation. Indeed in nuclear matter at low density, EDF approaches naturally predict clusterisation as mentioned above $[12,13]$. In finite rotating nuclei, clusterised states are predicted as a linear alpha chain in ${ }^{16} \mathrm{O}$ [23] (Fig. 2) or a ten alphas torus configuration in ${ }^{40} \mathrm{Ca}$ [24].

This introduces a new view of nuclear clustering: an hybrid phase between the quantum liquid one and the crystal phase [25]. This is understood from the ratio of the nucleon dispersion to the internucleon distance, the so-called localisation parameter. When this ratio is large, the nucleus behaves as a quantum liquid, as discussed above. When it is small, the system behaves as a crystal. Cluster states 


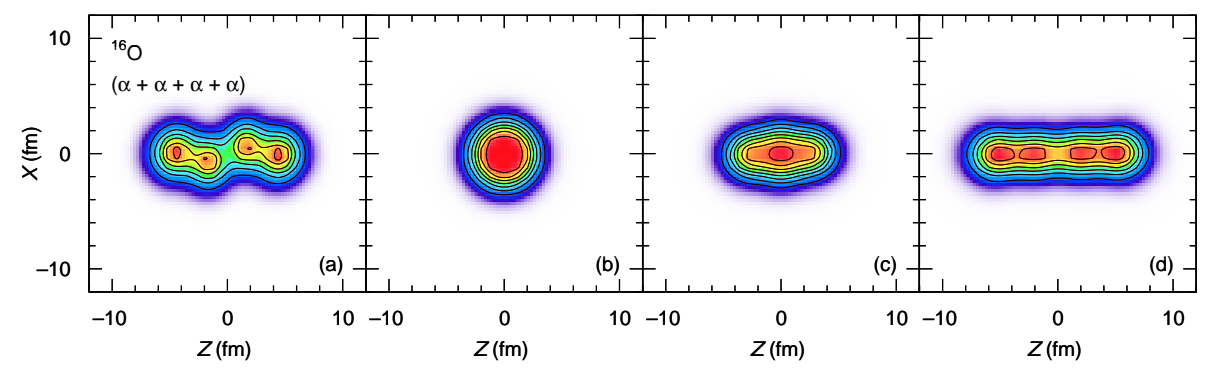

Figure 2. (color online). Total nucleon density distribution calculated using the cranking method for (a) the initial wave function, (b) the ground state, (c) the quasistable state, and (d) the four-alpha linear chain state. Reprinted figure with permission from T. Ichikawa et al., Phys. Rev. Lett. 107, 112501 (2011). Copyright (2011) by the American Physical Society.

are in between [26]. The localisation parameter allows to take into account finite size effects in nuclei whereas the quantality parameter discussed by Mottelson [15] is valid for infinite systems. Figure 3 displays the evolution of the localisation parameter as a function of the mass number, obtained both in a simple harmonic oscillator picture and with recent EDF calculations. Cluster states appear for a ratio close to one, which is for light nuclei. Heavy nuclei more behave as quantum liquid, and crystal nuclear states cannot occur in nuclei. It should be noted that such a quantum liquid-cluster-crystal transition can occur in the crust of neutron stars, which is a gravitationally constrained environment [6].

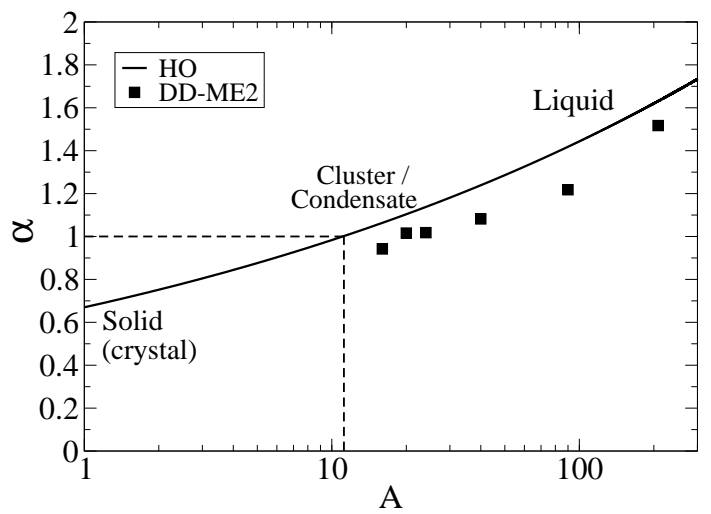

Figure 3. The localization parameter $\left(\alpha \equiv \frac{b}{r_{0}} \simeq \frac{\sqrt{\hbar R}}{r_{0}\left(2 m V_{0}\right)^{1 / 4}}\right.$ where $\mathrm{R}$ is the nuclear radius and $\mathrm{V}_{0}$ the depth of the confining nuclear potential) as a function of the number of nucleons (solid line). The average values of this parameter for ${ }^{16} \mathrm{O},{ }^{20} \mathrm{Ne},{ }^{24} \mathrm{Mg},{ }^{40} \mathrm{Ca}$, and ${ }^{90} \mathrm{Zr}$, calculated for the microscopic self-consistent solutions obtained using the functional DD-ME2, are denoted by squares. Reprinted figure with permission from J.-P. Ebran et al., Phys. Rev. C 87, 044307 (2013). Copyright (2013) by the American Physical Society. 
INPC 2013

\section{Nuclear dynamics}

Nuclear excitations are also described by EDF approaches. During the last decades there have been a continuous improvement of the EDF based method to microscopically describe nuclear excitations: doubly magic nuclei in the 70's [27, 28] (with the so-called RPA), semi-magic nuclei in the 2000's [29-31] (by including pairing correlations) and a few years later the even-even nuclei of the nuclear chart (by including deformation) [32-34]. Over the last years further improvements have been performed. For instance both the first $2^{+}$energies and their transition probabilities are well described in the deformed $\mathrm{Mg}$ isotopes using a beyond-mean-field model [35].

Improvements have also been performed in the description of the nuclear dipole response, which is the benchmark of the collective nuclear excitations. A very good agreement with the data on ${ }^{208} \mathrm{~Pb}$ is obtained using beyond RPA approaches [36, 37], such as phonon coupling, which allow to also predict the width of the nuclear response: the standard RPA approach only takes into account 1particle-1hole correlations, whereas more sophisticated approaches deal in addition with 2particles2 holes states or phonon coupling effects. Soft modes are also investigated. The so-called soft dipole mode, located at low energy, exhibits a rather non-universal pattern: it depends on the considered nucleus and also on the energy area: the low energy tail of the giant dipole resonance does not exhibit a similar structure than at lower energies. Several state-of-the-art calculations [38, 39] show that the picture is more complicated than the simple pygmy picture of an oscillating neutron skin. It should be noted that this discussion has been trigerred by precise measurements obtained on the low energy part of the dipole response in stable nuclei [40]. There are also recent EDFs studies of the soft modes in other multipolarities: the low energy quadrupole collectivity has been studied in [41] whereas a soft non-collective monopole mode has been predicted in Ni isotopes [42].

Recently is has been shown that the dipole polarisability (how the energy of the nucleus changes in an external field) is correlated to the dipole response, the neutron skin and the slope of the EoS symmetry energy $[43,44]$. Several recent works also relate the neutron skin and the density dependence of the symmetry energy $[45,46]$. Other works relate the (soft) dipole response to the density dependence of the symmetry energy [56]. There is therefore a "neutron-rich" triangular relation with three related quantities: i) the dipole response and the polarisability, ii) the density dependence of the symmetry energy of the equation of state and iii) the neutron skin. i) are measurable quantities, ii) should be carefully predicted for applications in neutrons stars for instance, and iii) is also predicted, although it recently started to become a direct measurable quantity with the recent PREX results [48]. There is therefore an intense activity around this "neutron-rich" triangle.

More exotic modes are also predicted by EDF based methods, in agreement with the data. Tidal waves are generated in a nucleus when its deformation increases with increasing angular momentum, keeping its angular frequency constant. This effect has been successfully described with the rotational data collected in ${ }^{102} \mathrm{Pd}$ [49]. Similarly, the anti-magnetic rotation has been evidenced in ${ }^{105} \mathrm{Cd}$ [50]. In such a mode, the angular momentum is generated by three loops of nucleonic currents, where two are anti-aligned. These results show the ability of EDF methods to predict complex and varied excited patterns, with a successful description of the data. More generally exotic excited modes are a rather dynamic and predictive sector in nuclear physics.

Turning to radioactive decays, recent results have been obtained in the weak decay sector. The tensor term of the nuclear interaction has been thoroughly discussed in the previous decade [51]. The tensor term is expected to have an important role in the beta decay because of its interplay between the neutron and proton orbits. Recent EDF-based predictions show that the tensor term is a crucial effect to accurately describe beta decay half lives on various neutron-rich doubly magic nuclei over the nuclear chart [52] (Fig. 4). Also, the neutrinoless double beta decay requires nuclear matrix 
elements for deformed nuclei, which are now very precisely predicted by beyond-EDF methods, using projection techniques [53].

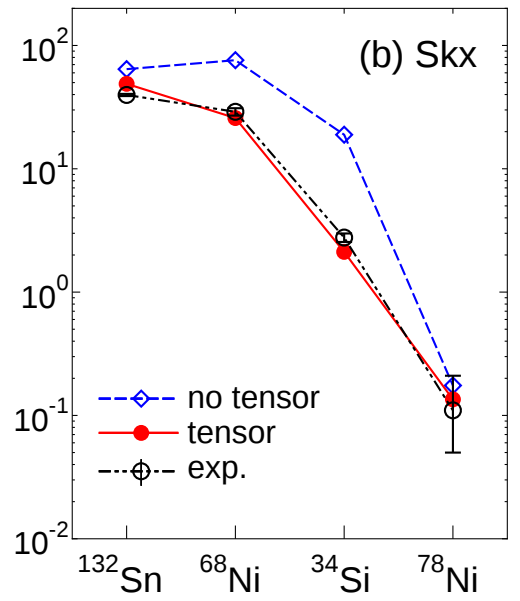

Figure 4. Beta-decay half-lives with and without the tensor force of ${ }^{132} \mathrm{Sn},{ }^{68} \mathrm{Ni},{ }^{34} \mathrm{Si}$, and ${ }^{78} \mathrm{Ni}$ calculated with the Skx EDF. The vertical axis is given in the unit of seconds. Reprinted figure with permission from F. Minato and C.L. Bai, Phys. Rev. Lett. 110, 122501 (2013). Copyright (2013) by the American Physical Society.

Finally, recent progress have been made in reaction mechanisms, directly described from nuclear structure EDF-based methods. This effort goes towards unified reaction models having the same input for the structure and reaction parts. For instance the two neutron transfer cross section from ${ }^{11} \mathrm{Li}$ is well described using Feynman diagrams from phonon coupling models together with superfluidity effects [54]. It should be noted that the magnitude of the cross sections is well reproduced, which is known to be a difficult task in two particle transfer calculations. Also time dependent EDF approaches allow to directly describe reactions mechanisms such as transfer one [55], which is promising for the unification of structure and reaction approaches.

\section{Conclusion}

In summary EDF methods have released significant results over the last years, moving towards a global description of the rich variety of the nuclear phenomenon: interesting paths are drawn for an universal description of quantum liquid and cluster states, and also an universal description of reaction and structure mechanisms. Interesting links are studied in the EoS in relationship with nuclear observables. The EDF reliability has been improved, allowing to draw the limits of the nuclear landscape. Accurate descriptions of exotic excitations, and of weak decay mechanisms are also available.

Technical terms have been avoided as much as possible is this report in order to focus on the nucleus' phenomenology. Technical improvements are of major importance because they are feeding the above mentioned achievements. One could mention for instance density matrix expansion in the density functional theory framework [56], projection techniques [57] or the treatment of three body effects $[58,59]$. They shall provide a new generation of EDF-models in order to further understand the rich nuclear phenomenology. 


\section{References}

[1] P. Hohenberg and W. Kohn, Phys. Rev. 136, B864 (1964).

[2] W. Kohn and L. J. Sham, Phys. Rev. 140, A1133 (1965).

[3] J. Engel, Phys. Rev. C 75, 014306 (2007).

[4] M. Bender, P.-H. Heenen, P.-G. Reinhard, Rev. Mod. Phys. 75, 121 (2003).

[5] D. Vretenar et al., Phys. Rep. 409, 101 (2005).

[6] J.M. Lattimer and M. Prakash, Phys. Rep. 333, 121 (2000).

[7] A.W. Steiner, this volume.

[8] E. Khan, J. Margueron and I. Vidaña, Phys. Rev. Lett. 109, 092501 (2012).

[9] J. Piekarewicz, Phys. Rev. C 83, 034319 (2011).

[10] M. Dutra et al., Phys. Rev. C 85, 035201 (2012).

[11] J.B. Natowicz et al., Phys. Rev. Lett. 104, 202501 (2010).

[12] M. Girod and P. Schuck, to be published in Phys. Rev. Lett. (2013).

[13] J.-P. Ebran Private Communication (2013).

[14] A. Rios and V. Soma, Phys. Rev. Lett. 108, 012501 (2012).

[15] B. Mottelson, Nuclear Structure, Les Houches, Session LXVI, 25 (1996).

[16] D. Lunney et al., Proc. of International Conference on Exotic Nuclei and Atomic Masses (ENAM

95), Arles, France, June 19-23, 1995.

[17] J. Erler et al., Nature 486, 509 (2012).

[18] M. Kortelainen et al., Phys. Rev. C 85, 024304 (2012).

[19] L. Li et al., Phys. Rev. C 85, 024312 (2012).

[20] Y. Shi et al., Phys. Rev. Lett. 108, 092501 (2012).

[21] V. Prassa et al., Phys. Rev. C 86, 024317 (2012).

[22] K. Nomura et al., Phys. Rev. Lett. 108, 132501 (2012).

[23] T. Ichikawa et al., Phys. Rev. Lett. 107, 112501 (2011).

[24] T. Ichikawa et al., Phys. Rev. Lett. 109, 232503 (2012).

[25] J.-P. Ebran et al., Nature 487, 341 (2012).

[26] J.-P. Ebran et al., Phys. Rev. C 87, 044307 (2013).

[27] G.F. Bertsch and S.F. Tsai, Phys. Rep. 18, 125 (1975).

[28] K.F. Liu and Nguyen Van Giai Phys. Lett. B 65 (1976) 23.

[29] E. Khan and Nguyen Van Giai, Phys. Lett. B 472, 253 (2000).

[30] G. Colò et al., Phys. Lett. B 485, 362 (2000).

[31] N. Paar et al., Phys. Rev. C 67, 034312 (2003).

[32] D. Pena Arteaga and P. Ring Phys. Rev. C 77034317 (2008).

[33] S. Péru and H. Goutte, Phys. Rev. C 77, 044313 (2008).

[34] K. Yoshida and N. Van Giai, Phys. Rev. C 78, 014305 (2008).

[35] N. Hinohara et al., Phys. Rev. C 84, 061302(R) (2011).

[36] N. Lyutorovich et al., Phys. Rev. Lett. 109, 092502 (2012).

[37] E. Litvinova, P. Ring and V. Tselyaev, Phys. Rev. Lett. 105, 022502 (2012).

[38] D. Gambacurta, M. Grasso and F. Catara, Phys. Rev. C 84, 034301 (2011).

[39] M. Martini, S. Péru and M. Dupuis, Phys. Rev. C 83, 034309 (2011).

[40] J. Endres et al., Phys. Rev. Lett. 105, 212503 (2010).

[41] C. Walz et al., Phys. Rev. Lett. 106, 062501 (2011).

[42] E. Khan, N. Paar and D. Vretenar, Phys. Rev. C 84, 051301 (2011). 
[43] J. Piekarewicz et al., Phys. Rev. C 85, 041302(R) (2012).

[44] P.-G. Reinhard and W. Nazarewicz, Phys. Rev. C 81, 051303 (2010).

[45] B.K. Agrawal, J.N. De and S.K. Samaddar, Phys. Rev. Lett. 109, 262501 (2012).

[46] X. Roca-Maza et al., Phys. Rev. Lett. 106, 252501 (2011).

[47] A. Carbone et al., Phys. Rev. C 81, 041301(R) (2010).

[48] S. Abrahamyan et al., Phys. Rev. Lett. 108, 112502 (2012).

[49] A.D. Ayangeakaa et al., Phys. Rev. Lett. 110, 102501 (2013).

[50] P.W. Zhao et al., Phys. Rev. Lett. 107, 122501 (2011).

[51] T. Otsuka, T. Matsuo and D. Abe, Phys. Rev. Lett. 97, 162501 (2006).

[52] F. Minato and C.L. Bai, Phys. Rev. Lett. 110, 122501 (2013).

[53] T.R. Rodriguez and G. Martinez-Pinedo, Phys. Rev. Lett. 105, 252503 (2010).

[54] G. Potel et al., Phys. Rev. Lett. 105, 172502 (2010).

[55] C. Simenel, Phys. Rev. Lett. 105, 192701 (2010).

[56] B.G. Carlsson and J. Dobaczewski, Phys. Rev. Lett. 105, 122501 (2010).

[57] G.F. Bertsch and L.M. Robledo, Phys. Rev. Lett. 108, 042505 (2012).

[58] T. Otsuka et al., Phys. Rev. Lett. 105, 032501 (2010).

[59] A. Gezerlis and G.F. Bertsch, Phys. Rev. Lett. 105, 212501 (2010). 